\title{
A fuzzy TOPSIS based analysis toward selection of effective security requirements engineering approach for trustworthy healthcare software development
}

\author{
Md Tarique Jamal Ansari ${ }^{1^{*}}$, Fahad Ahmed Al-Zahrani ${ }^{2}$, Dhirendra Pandey ${ }^{1}$ and Alka Agrawal ${ }^{1}$
}

\begin{abstract}
Background: Today's healthcare organizations want to implement secure and quality healthcare software as cybersecurity is a significant risk factor for healthcare data. Considering security requirements during trustworthy healthcare software development process is an essential part of the quality software development. There are several Security Requirements Engineering (SRE) methodologies, framework, process, standards available today.

Unfortunately, there is still a necessity to improve these security requirements engineering approaches. Determining the most suitable security requirements engineering method for trustworthy healthcare software development is a challenging process. This study is aimed to present security experts' perspective on the relative importance of the criteria for selecting effective SRE method by utilizing the multi-criteria decision making methods.
\end{abstract}

Methods: The study was planned and conducted to identify the most appropriate SRE approach for quality and trustworthy software development based on the security expert's knowledge and experience. The hierarchical model was evaluated by using fuzzy TOPSIS model. Effective SRE selection criteria were compared in pairs. 25 security experts were asked to response the pairwise criteria comparison form.

Results: The impact of the recognized selection criteria for effective security requirements engineering approaches has been evaluated quantitatively. For each of the 25 participants, comparison matrixes were formed based on the scores of their responses in the form. The consistency ratios (CR) were found to be smaller than $10 \%(C R=9.1 \%<$ 10\%). According to pairwise comparisons result; with a 0.842 closeness coefficient (Ci), STORE methodology is the most effective security requirements engineering approach for trustworthy healthcare software development.

Conclusions: The findings of this research study demonstrate various factors in the decision-making process for the selection of a reliable method for security requirements engineering. This is a significant study that uses multicriteria decision-making tools, specifically fuzzy TOPSIS, which used to evaluate different SRE methods for secure and trustworthy healthcare application development.

Keywords: Security requirements, Software security, Healthcare application, Quality software development, Fuzzy TOPSIS, Risk analysis

\footnotetext{
*Correspondence: titjansari@gmail.com

'Department of Information Technology, Babasaheb Bhimrao Ambedkar University, Lucknow, Uttar Pradesh, India

Full list of author information is available at the end of the article
}

(c) The Author(s). 2020 Open Access This article is licensed under a Creative Commons Attribution 4.0 International License, which permits use, sharing, adaptation, distribution and reproduction in any medium or format, as long as you give appropriate credit to the original author(s) and the source, provide a link to the Creative Commons licence, and indicate if changes were made. The images or other third party material in this article are included in the article's Creative Commons licence, unless indicated otherwise in a credit line to the material. If material is not included in the article's Creative Commons licence and your intended use is not permitted by statutory regulation or exceeds the permitted use, you will need to obtain permission directly from the copyright holder. To view a copy of this licence, visit http://creativecommons.org/licenses/by/4.0/ The Creative Commons Public Domain Dedication waiver (http://creativecommons.org/publicdomain/zero/1.0/) applies to the data made available in this article, unless otherwise stated in a credit line to the data. 


\section{Background}

Today's Information Technology (IT) has had a massive impact on a number of areas of society. We live in an era of IT in which technological resources such as software, hardware, and sensors are becoming an essential accessory in our daily lives $[1,2]$. As we are increasingly dependent on software in our day-to-day life, therefore this technological dependency is a growing demand for secure software. Traditionally, software vendors have focused on improving the quality of software code to improve software security and quality [3]. Currently, security and privacy has become an emerging subject than ever between many healthcare organization, researchers, IT professionals, primarily due to the recent increase of Ransomware attacks around the world, and moreover due to the increasing amount of data and the exponential growth involved with the network of systems and technologies that produces and manipulates it. Unfortunately, attackers know the importance of data in patient medicinal records, rendering healthcare the biggest priority for cyber threats on ransomware comparative to any other sector.

The security violation for a healthcare software product causes enormous fatalities. For this reason, it is necessary to develop such a security-critical software system in the best conceivable way. It does not inevitably mean absolute security, however a reasonable highsecurity level in relation to the given limitations. In recent years, literature has offered a number of security and privacy requirements engineering methods that assist the software system designers and developers to implement security and privacy concerns presented in the traditional development model. Several methods deliberate security or privacy requirements independently, however, some other approaches consider privacy as a subset of security [4]. Software security requirements have become an important part of the overall requirements analysis process during the software development process.

In the United States in 1996 the Health Insurance Portability and Accountability Act, known as HIPAA, was enacted. The law sets standards for data security as well as privacy to safeguard patient records. HIPAA compliance has now become an important consideration in the healthcare industry for software engineers in recent times, as several high-profile data attacks have exposed millions of medical information nationally [5]. According to HIPAA Journal, there were 3054 data breaches in the healthcare industry affecting over 500 documents between 2009 and 2019. All these violations resulted in the destruction, theft, disclosure, or unauthorized release of 230,954,151 data in healthcare organizations. That is comparable to much more than $69.78 \%$ of the United States population. Data breaches of healthcare information were confirmed at a frequency of
1.4 per day in 2019 [6]. The following Fig. 1 shows the year-wise number of healthcare data breaches.

Software security is the fastest growing paradigm in the IT security field $[7,8]$. According to the recent IDC Worldwide Semiannual Security Spending Guide, expenditure on security hardware, services, and software in Asia/Pacific is expected to reach USD 16.4 billion in 2019 , an increase of $20.01 \%$ over the previous year. Further, the IDC assumes investment in software securityrelated products and services to grow at a five-year compound annual growth rate (CAGR) of $20.1 \%$ over the forecast period (2018-23) and reach USD 34 billion by 2023 [9].

Security requirements are one of the most important parts of all non-functional requirements. Negotiation with software security requirements during software development may result in disastrous failure of the software product affecting enormous damage of valuable assets. Special attention should be given to the security requirements of the software product during the software development as a software system does not exist physically.

The introduction of electronic health records raises a variety of problems related to protection and privacy that need addressing. Cyber-security in the healthcare sector has been one of the major challenges. Recent initiatives to digitize various dimensions of healthcare, the transition to electronic health, would have a huge effect on healthcare sector growth. Healthcare records and information are distributed across networks, which mean they are sensitive and vulnerable to a range of security breaches. The possible threats for healthcare breaches may include medical staff, malware or phishing attacks, healthcare suppliers, electronic health equipment, unauthorized access, etc. [5]. There are a variety of reasons for the targeted healthcare records. Several flaws in healthcare technology are available that can damage confidential healthcare data. Electronic medical records with other sensitive information can easily fall into fraudulent hands, despite proper monitoring. Huge amounts of confidential data and inadequate protection appear to be key factors, however the high value and reliability of the information is actually what encourages most attackers. Healthcare includes the highest proportion of weak authentication implementations and the closest frequency of data leakage. Such data points are also problematic considering the amount of healthcare services that interact with confidential patient data.

Obviously, the biggest challenge is to avoid improper access or misuse of the medical records. The next challenge is whether any permitted access is traceable. This in effect includes procedures for safe identification of individuals including doctors, patients, labs, etc. Eventually, documents in a censored or anonymized form 


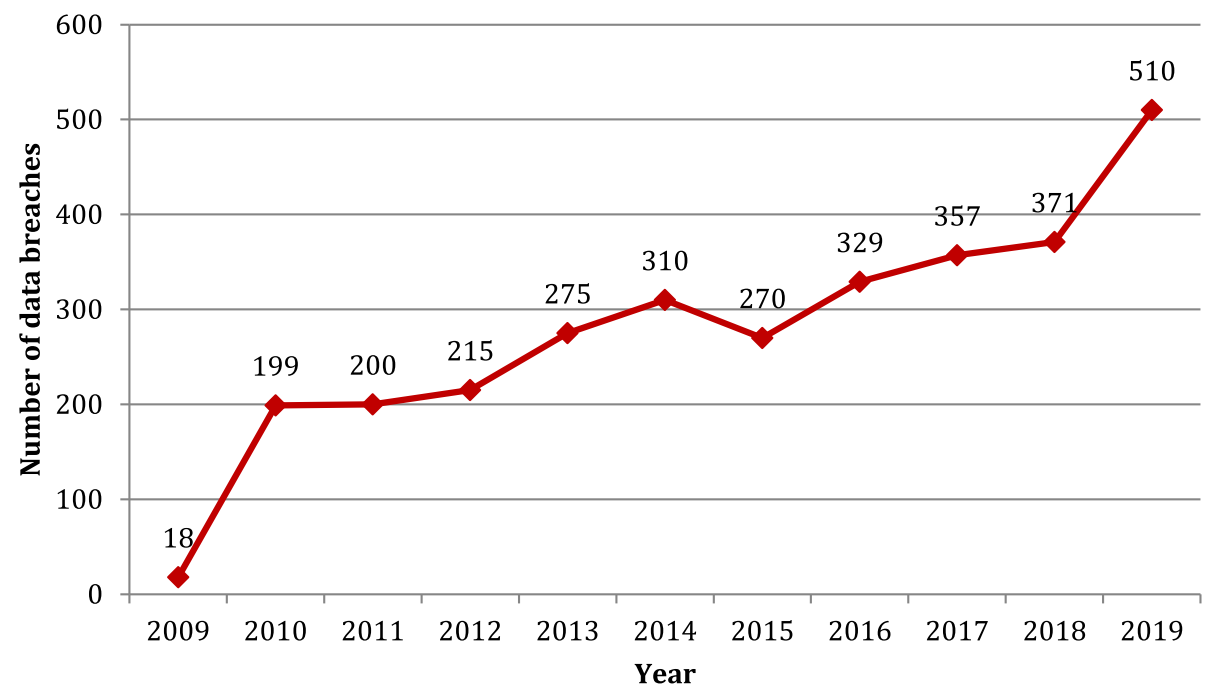

Fig. 1 Year-wise healthcare data breaches (Source: HIPAA Journal)

ought to be made accessible to third parties. The rising threat to healthcare and patient's confidential data enables application developers to become more rigorous in designing successful security requirements to make trustworthy healthcare web applications. For many healthcare application developers, security requirements are a major concern, and some of those have vital role in the proper organizational goals of the software development will try to incorporate security into the application development process as soon as possible.

The identification of threats is significant and it facilitates the development of realistic and relevant security requirements. Implementations for healthcare services should be capable of storing and handle claims for refund. In addition, the database would be open to enable access to information about patient treatment in the event of immediate necessity. There's really common understanding between many practitioners and researchers that security effective and efficient security requirements elicitation is important. Therefore the creation of healthcare applications requires the integration of functional, non-functional requirements and also architectural engineering practices [10]. One challenge to resolve is the significant advancements in the market needs, including during the creation of healthcare applications. Holding the emphasis on business properties, though, which tend to be much more constant, is an incentive for IT system growth to be better matched with the company. For a healthcare software application, assets are something that are confidential and has financial value to the organization, and that this is central to achieving its strategic goals. It is important to keep them protected. Figure 2 illustrates various types of assets for the healthcare application system. Business assets are patient confidential data, administration of healthcare authorizations, and patient's individual records in healthcare industry, while the IT asset includes different hardware, software, associated stakeholders and networks. A web based healthcare application should properly incorporate all the effective security requirements in order to deliver trustworthy healthcare application software.

We notice that different security requirements engineering techniques are available for the elicitation of security requirements in order to develop a quality and trustworthy healthcare software system. However, the selection of the most appropriate SRE approach for trustworthy healthcare software development is a challenging task. The objective of this paper is to identify the different criteria for the analysis of different security requirements engineering approaches. Further, we select the ISO 27005 standard [8] criteria for the selection of effective security requirements engineering approach. We evaluate each criterion by itself or in comparison with other requirements, while ignoring the relationships existing between them, and also without regard to the effect of others on this priority value. In this paper, the researchers formally review the different security requirements engineering approaches and identify five best approaches as alternatives for comparative analysis. The main purpose of prioritizing existing security requirements engineering approaches is to help in quality and trustworthy software development. In order to improve decision making and to achieve this, it is necessary to establish a process adapted to requirements that take into account these relationships, to help provide consistency to the prioritization done. For that, before assigning a final value of priority to each criterion it is important to consider the operational significance of those criteria in 


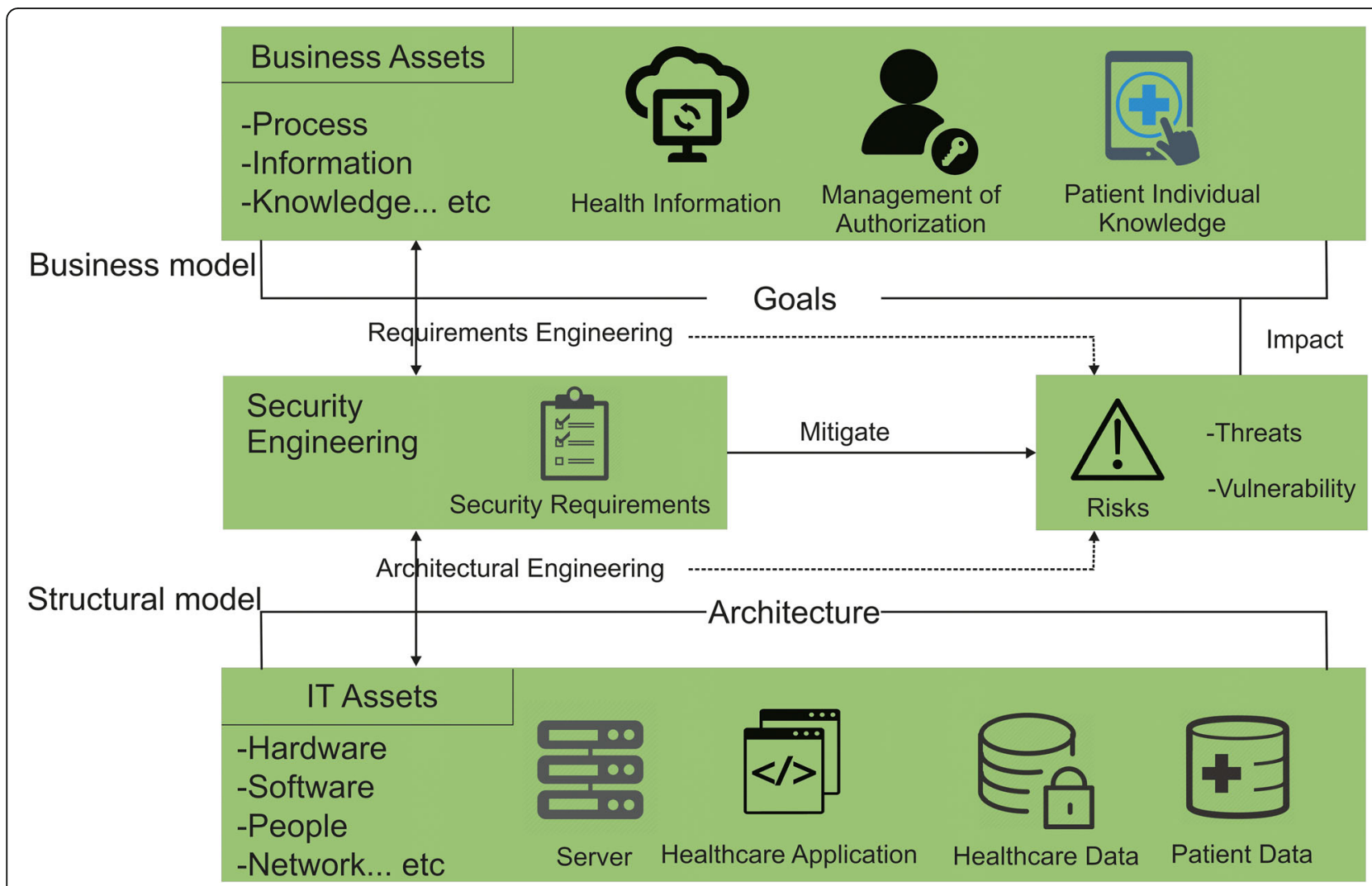

Fig. 2 Security requirements engineering for trustworthy healthcare system

the context of effective security requirements engineering with which it is in interdependency. In this paper we use the principle of pair-wise comparisons of fuzzy TOPSIS [11] method that is deliberated as the most helpful method, to help to accomplish the best decisions conceivable and to clearly present the rationality of the decision made about prioritization.

\section{Security requirements engineering}

Security requirements engineering is an area of software engineering, which comprises security, safety, risk, vulnerabilities, and mitigation mechanisms. Security requirements engineering has over the years proven to be a challenging task. This is especially the case because pinpointing what security requirements are having been difficult. Despite all these challenges, the demand for developing security requirements elicitation methods for the changing requirements of networked environments is great. Mellado et al. [12] describe software security engineering as a practice through which to address software security issues in a systematic manner, is known to be a very important part of the software development process for the achievement of secure software systems. According to Devanbu \& Stubblebine security requirement is considered as a manifestation of a high-level organizational policy into the detailed requirements of a specific system [13]. Lee et al. [14] draw attention to the significance of considering security requirements in the development life cycle, but do not define them. After analyzing the existing literature and best practices in the area of software security engineering, the authors have defined the security requirements engineering as:

"Security requirements engineering is a process of generating prerequisite non-functional requirements that stipulate a compulsory amount of system quality to prevent the adversary's attack. It should be considered early during the software development process to deliver a trustworthy software product."

Software security engineering procedure should include the use of repeatable and organized processes to guarantee that the set of requirements found is complete, reliable, easy to recognize and analyzable by the different participants involved in the software development process [15]. Security needs to be considered as a quality constraint in all the phases of software development process [16]. To develop a security-critical software system [17] many security requirement frameworks have been developed by different authors. Some of the famous security requirements engineering approaches are STORE [2], MOSRE [18], SREF [19], SREP [20], SQUARE [21]. Security requirements engineering is an 
important activity since bad security requirements can lead to ineffective security or worth security holes [22]. The following section briefly discusses each security requirements engineering approach.

\section{SREP}

Mellado et al. proposed Security Requirements Engineering Process (SREP) [20] is a method that focuses on the Common Criteria security assessment standard [23] and is considered the principle of reuse. It deals systematically and intuitively with the security requirements. This approach offers a catalog of security resource management and synchronizes the Common Criteria into application development life cycle, in order to unify the notions of requirements engineering as well as security engineering. Several definitions and methods have been used in consideration of this method: a catalog of security tools (with properties, threats, specifications, etc.), cases of abuse, threat / attack trees, including cases of security uses. SREP was designed based on an understanding of the ISO / IEC 27002 standard [20].

SREP approach includes the following steps:
I. Agree on Definitions
II. Identify Vulnerable \&/or Critical Assets
III. Identify Security Objectives \& Dependencies
IV. Identify Threats \& Develop Artifacts
V. Risk Assessment
VI. Elicit Security Requirements
VII. Categorize \& Prioritize Requirements
VIII.Requirement Inspection
IX. Repository Improvement

\section{SQUARE}

SQUARE (Security Quality Requirements Engineering) [21] developed by Carnegie Mellon University, It is a 9step method aimed at categorizing and prioritizing the criteria for protection. This method provides a way to solicit, categorize, and prioritize security specifications for software applications. This methodology focuses on building features into the early stages of a software development lifecycle. It may also be effective to document and analyse the safety aspects of drafted applications and future changes may be guided and improvements to those structures. Every step is designated with inputs, outputs, members and procedures:

I. Agree on definitions

II. Identify security goals

III. Develop Artifacts to support security requirements definition

IV. Perform risk assessment

V. Select elicitation techniques

VI. Elicit security requirements
VII. Categorize requirements

VIII.Prioritize requirements

IX. Requirements inspection

The SQUARE method had been modified to handle privacy (P-SQUARE) as well as acquisition (A-SQUARE) explicitly.

\section{STORE}

Ansari et al. developed Security Threat Oriented Requirements Engineering Methodology (STORE) Methodology which is a ten-step security threat centric security requirements engineering methodology [2]. It provides methodological standards for organizational security systems with the help of standard system platforms as well as interfaces in the perspective of increased possible key infrastructure safety risks. The following section lists all the steps of STORE methodology as well as describing the functionality of each step.

I. Identify System Goals

II. Identify and Prioritize Stakeholders

III. Agreed upon Goals

IV. Asset Identification

V. Security Attack Analysis

- Point of Attack (PoA)

- Point of Belief (PoB)

- Point of Conjecture (PoC)

- Point of Dependency (PoD)

VI. Threat Identification and Categorization

VII. Risk Evaluation and Prioritization

VIII.Security Requirements Elicitation

IX. Security Requirements Validation

X. Security Requirements Specification Document

\section{MOSRE}

The Model Oriented Security Requirements Engineering approach [18] seeks to use different models such as application use cases, a misuse case etc. to enable quality and safety and requires analysis. It is designed to refer to web based application development. The peculiarity of MOSRE is that it includes the verification of objectives for whole system, the identification and modeling of non-security requirements prior to actually addressing the security requirements. It is therefore a technique that can be implemented to the entire phase of requirements engineering, with special emphasis on safety. Different stages of MOSRE framework are:

I. Inception: Recognize web app goals, stakeholders as well as assets

II. Elicitation

III. Elaboration: Produce structural analysis models

IV. Negotiation and validation of requirements 


\section{SREF}

Haley et al. proposed Security Requirements Engineering Framework [19] which is a combination of engineering requirements as well as security requirements. This is recursive as it reverts back and forward between design and technical specifications. The SREF approach provides following four steps:

I. Identify functional requirements

II. Identify security goals

- Identify assets

- Generate threat description

- Apply management principles (separation of duties, functions, ..)

III. Identify security requirements: Constraints on some or all of the security objectives. The safety requirements are descriptively represented.

IV. Construct satisfaction arguments: represent that the application is capable of meeting security requirements.

\section{Methods}

To make good decisions we must well prioritize the recognized ISO 27005 standard criteria for effective security requirement engineering selection, and so it is important to take the consideration of all 25 security experts into account when prioritizing. In our prioritization process we use the technique of pairwise comparison of different criteria through fuzzy TOPSIS. In order to selection of best SRE approach; we need to compare pairs of these criteria.

\section{Criteria and alternatives selection}

The first step in this process is the establishment of a hierarchy model. The hierarchy model is composed of the seven criterion group Security goal, Security requirement, Stakeholder, Asset, Threat, Vulnerability, Risk. After the hierarchy has been established, the criteria must be evaluated in pairs so as to determine the relative importance between them and their relative weight to the global goal. The study was planned and conducted to comparatively evaluate the different criteria which are considered by the software developer during the selection of effective security requirements engineering approach. A survey form was prepared to determine the prioritization of the characteristics incorporated when choosing effective security requirements engineering approach for the development of trustworthy healthcare software system. This form was given to 25 security experts to collect their estimation on the pair-wise criteria comparisons and fuzzy TOPSIS model was created. A set of ISO 27005 standard criteria represents the balanced hierarchical structure consisting of the seven main criteria and five alternatives incorporating the objectives and criteria when choosing the effective alternative as a security requirements engineering approach. Some of the popular SRE approaches which are used in this study are SREP, SQUARE, STORE, MOSRE and SREF which are represented by A1, A2, A3, A4, and A5 respectively. The Fig. 3 shows the hierarchy representation of different criteria and alternatives.

We mailed pairwise questionnaire form based on seven points scale to each of the security experts. The security experts were given a questionnaire that contained a pairwise comparison sheet. The members consisted of 25 security experts who were serving in the different software organizations and who had experience exceeding 15 years having deep knowledge about security requirements engineering process during software development. All security experts were security domain experts who practice different security requirements in their working places to build quality software products and have valuable knowledge about the different modern threats and software attack mechanism. They responded about their satisfaction with their choices. Further the collected data analyzed separately for each security expert. The following Table 1 shows the detailed description of each criterion.

\section{Fuzzy TOPSIS method}

Many multi-criteria-decision-making (MCDM) methods were developed in order to rank alternatives differently. Although evaluations of alternatives offered by MCDM methods may sometimes be in contract, there are circumstances where distinct MCDM methods produce very different recommendations [24-26]. A finite number of possible outcomes are selected, prioritized and ranked by experts. Because there are several techniques involved, Hwang and Yoon also include taxonomy to identify the methods as: assessment metrics from experts, essential information attributes, and a big class of methods. Even the description gives us a simple path to learn MCDM approaches [11]. They introduced Technique for Order of Preference by Similarity to Ideal Solution (TOPSIS) which defines an instrument called correlation to the ideal-positive solution as well as disconnection from the ideal-negative. The technique then prefers an alternative with highest correlation to the ideal-positive solution. They presented the TOPSIS method built on the concept that even a significant short distance through an ideal solution ought to be the best option available. 


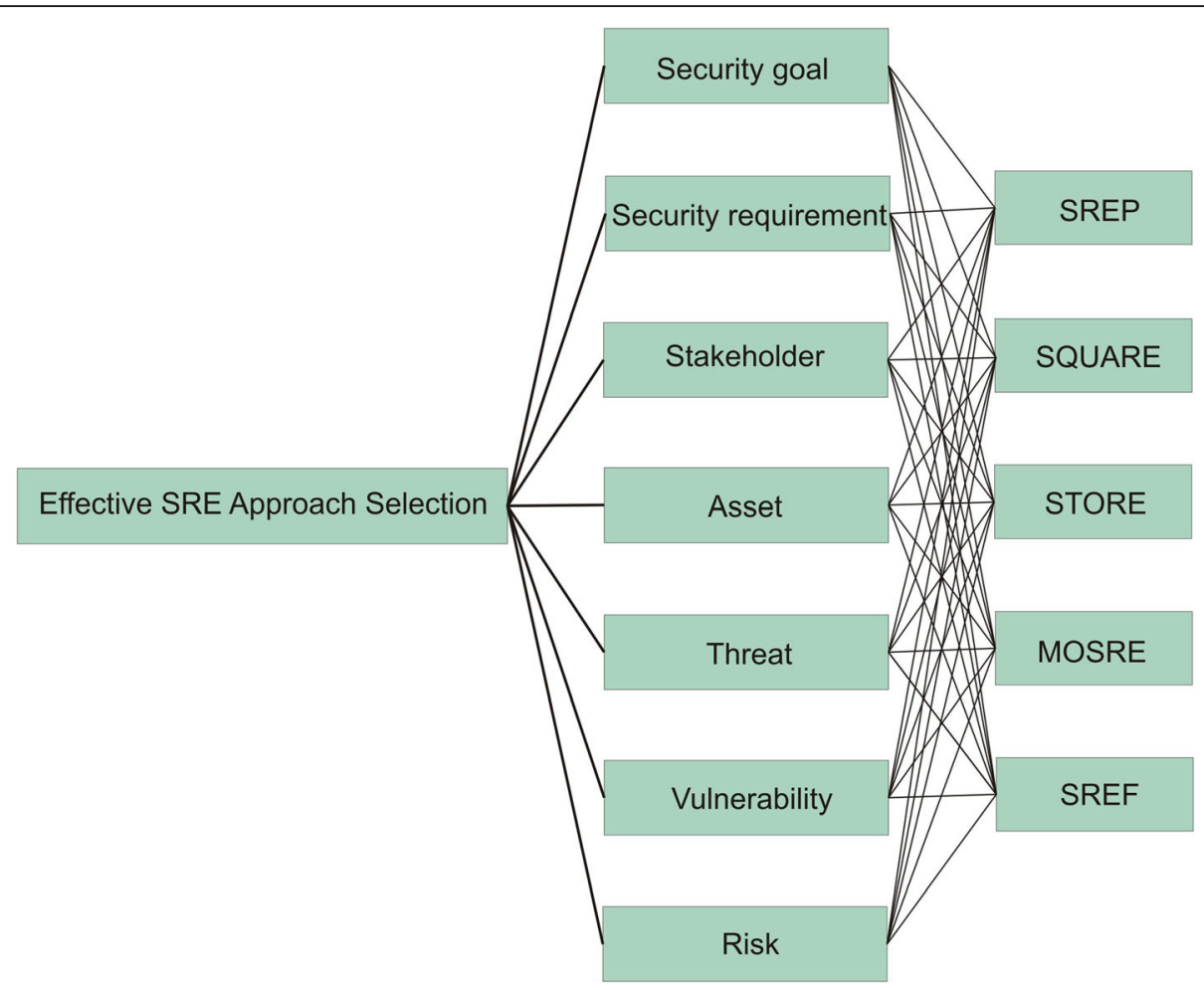

Fig. 3 Hierarchical model of criteria and alternatives

Several researches are available wherein the authors used the fuzzy TOPSIS method to develop effective decisions. Ashrafzadeh et al. [27] presented a Fuzzy TOPSIS method to multi-criteria decision making for choosing warehouse location under limited or imperfect data. They first defined the different selection criteria for the warehouse location and afterwards presented the alternative approaches against all the selected criteria with the help of domain experts with linguistic scale evaluations. Fuzzy TOPSIS is often used to produce selection of the best alternatives for aggregate scores. They also demonstrate a successful implementation of fuzzy TOPSIS to something like a true issue of selecting a big corporation's warehouse location in Iran. Sevkli et al. [28] used the fuzzy TOPSIS method to select suppliers at a Turkish

Table 1 The ISO 27005 standard criteria for effective SRE approach selection

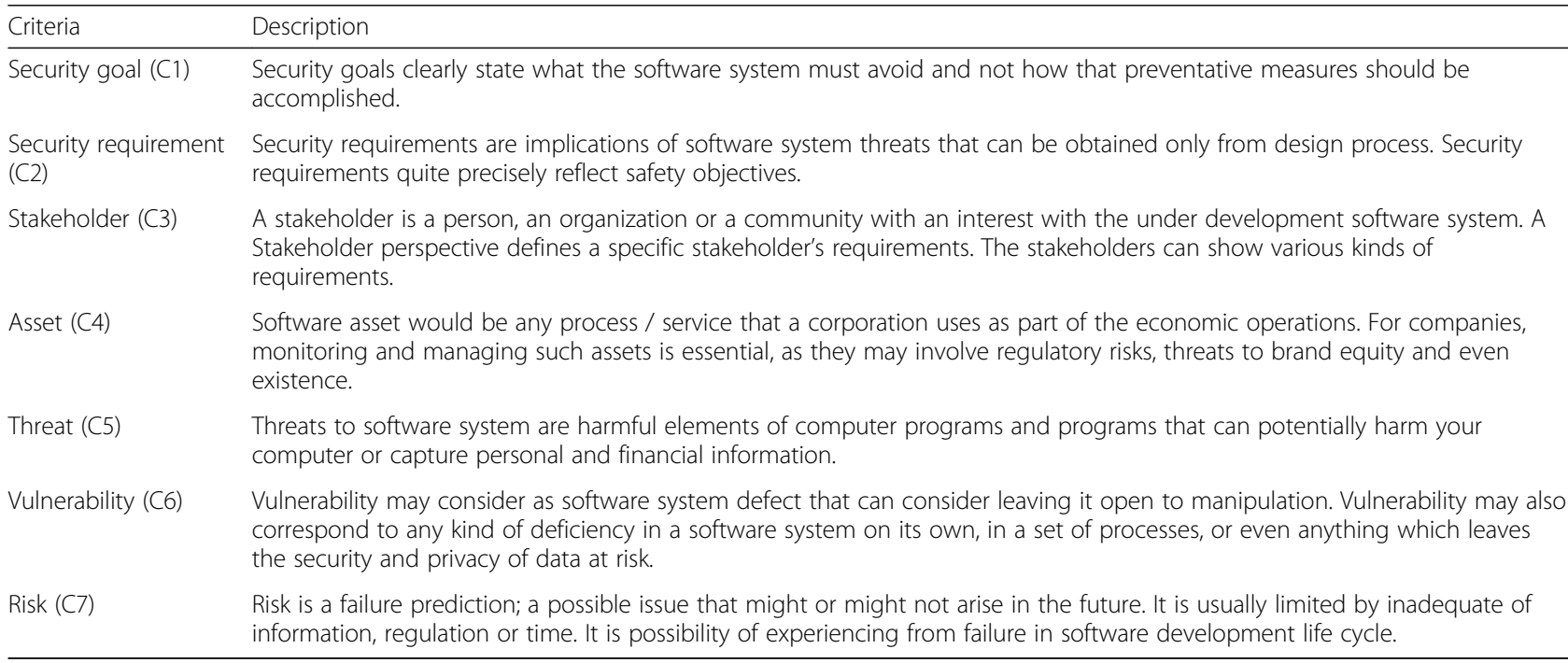




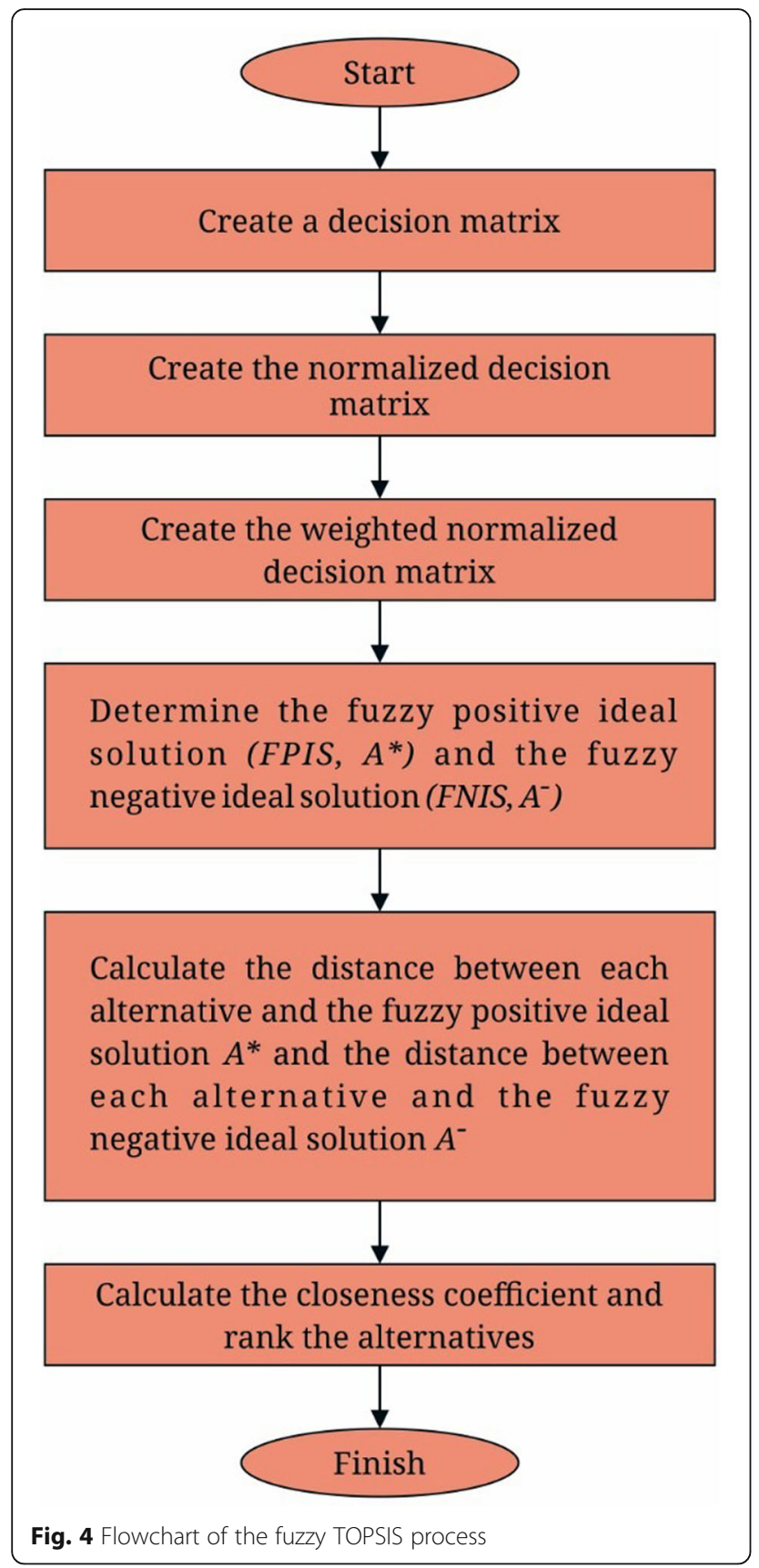

Table 2 Characteristics of Criteria

\begin{tabular}{llll}
\hline & Criteria & Type & Weight \\
\hline 1 & C1 & + & $0.143,0.143,0.143$ \\
2 & C2 & + & $0.143,0.143,0.143$ \\
3 & C3 & + & $0.143,0.143,0.143$ \\
4 & C4 & + & $0.143,0.143,0.143$ \\
5 & C5 & + & $0.143,0.143,0.143$ \\
6 & C6 & + & $0.143,0.143,0.143$ \\
7 & C7 & + & $0.143,0.143,0.143$ \\
\hline
\end{tabular}

Table 3 Fuzzy Scale

\begin{tabular}{lllll}
\hline Code & Linguistic terms & $\mathrm{L}$ & $\mathrm{M}$ & $\mathrm{U}$ \\
\hline 1 & Very low & 0 & 0 & 1 \\
2 & Low & 0 & 1 & 3 \\
3 & Moderately low & 1 & 3 & 5 \\
4 & Moderate & 3 & 5 & 7 \\
5 & Moderately high & 5 & 7 & 9 \\
6 & High & 7 & 9 & 10 \\
7 & Very high & 9 & 10 & 10 \\
\hline
\end{tabular}

manufacturing industry. They applied this method to assess the performance of alternatives on the shopping site and rank the primary importance appropriately for each other. TOPSIS considers a MADM issue as a geometric structure with $m$ points in $n$ dimensional space, with $\mathrm{m}$ alternatives. The approach is based on the principle that the selected alternative should be the quickest range from the ideal-positive solution as well as the longest range from the idealnegative.

In this method if each characteristic takes on asymptotically raising or lowering variation, then maybe an ideal solution can be easily defined. That solution consists of all possible to achieve best attributes, since the worst solution consists of all attainable worst attribute values. Assumed a decision-making issue with multiple criteria has $\mathrm{n}$ alternatives, $\mathrm{A} 1, \mathrm{~A} 2, \ldots, \mathrm{An}$ and $\mathrm{m}$ criteria, $\mathrm{C} 1, \mathrm{C} 2, \ldots, \mathrm{Cm}$. Each alternative is assessed against the criteria of $\mathrm{m}$. All the values/ratings are allocated to alternatives regarding decision matrix represented by $\mathrm{X}\left(\mathrm{x}_{\mathrm{ij}}\right)_{\mathrm{m} \times \mathrm{n}}$. Let $\mathrm{W}=\left(\mathrm{w}_{1}, \mathrm{w}_{2}, \ldots, \mathrm{w}_{\mathrm{m}}\right)$ be the weight vector of criteria, satisfying $\sum_{j-1}^{m} w_{j}=1$.

The fuzzy TOPSIS method comprises of the following steps as shown in Fig. 4.

\section{Step 1 create a decision matrix}

In this research study there are 7 criteria and 4 alternatives which are rated and ranked according to the FUZZY TOPSIS system. The following Table 2 describes the characteristics of criterion as well as weight assigned with each criterion.

The Table 3 below shows the seven point fuzzy scale used in this research study.

\section{Step 2 create the normalized decision matrix}

A normalized decision matrix can be estimated by the following relation based on the positive and negative ideal solutions: 
Table 4 Decision matrix

\begin{tabular}{llllllll}
\hline & C1 & C2 & C3 & C4 & C5 & C6 & C7 \\
\hline A1 & $3.480,5.480,7.440$ & $4.680,6.680,8.520$ & $5.080,7.000,8.520$ & $4.920,6.880,8.560$ & $5.080,7.000,8.480$ & $5.800,7.680,9.120$ & $5.240,7.160,8.720$ \\
A2 & $4.760,6.760,8.560$ & $5.320,7.280,8.880$ & $4.680,6.680,8.480$ & $5.560,7.560,9.080$ & $5.560,7.440,8.840$ & $5.160,7.040,8.560$ & $5.000,7.000,8.600$ \\
A3 & $4.840,6.800,8.480$ & $5.480,7.440,9.000$ & $5.320,7.280,8.920$ & $5.480,7.400,8.920$ & $5.400,7.280,8.840$ & $5.120,7.000,8.520$ & $5.240,7.160,8.800$ \\
A4 & $4.600,6.600,8.320$ & $5.160,7.080,8.640$ & $4.760,6.720,8.400$ & $4.680,6.640,8.360$ & $4.920,6.800,8.440$ & $4.680,6.680,8.480$ & $4.520,6.520,8.240$ \\
A5 & $4.680,6.680,8.520$ & $5.000,6.960,8.560$ & $5.160,7.120,8.760$ & $4.840,6.800,8.520$ & $4.680,6.680,8.480$ & $4.520,6.520,8.320$ & $4.360,6.360,8.200$ \\
\hline
\end{tabular}

$$
\begin{aligned}
& \tilde{r}_{i j}=\left(\frac{a_{i j}}{c_{j}^{*}}, \frac{b_{i j}}{c_{j}^{*}}, \frac{c_{i j}}{c_{j}^{*}}\right) ; c_{j}^{*}=\max _{i} c_{i j} ; \text { Positive ideal solution } \\
& \tilde{r}_{i j}=\left(\frac{a_{j}^{-}}{c_{i j}}, \frac{a_{j}^{-}}{b_{i j}}, \frac{a_{j}^{-}}{a_{i j}}\right) ; a_{j}^{-}=\min _{i} a_{i j} ; \text { Negative ideal solution }
\end{aligned}
$$

\section{Step 3 create the weighted normalized decision matrix}

Depending on different weights within each criterion, as per the following formula, the weighted normalized decision matrix can be determined by calculating the weight of each criterion in the standard fuzzy decision matrix.

$$
\tilde{\mathrm{v}}_{\mathrm{ij}}=\tilde{\mathrm{r}}_{\mathrm{ij}} \cdot \tilde{\mathrm{w}}_{\mathrm{ij}}
$$

Where $\tilde{w}_{i j}$ represents weight of criterion $c_{j}$

Step 4 determine the fuzzy positive-ideal solution (FPIS) $a^{*}$ and fuzzy negative-ideal solution (FNIS) A-

The FPIS and FNIS of the alternatives can be defined as follows:

$$
\begin{aligned}
& A^{*}=\left\{\tilde{\mathrm{v}}_{1}^{*}, \tilde{\mathrm{v}}_{2}^{*}, \ldots, \tilde{\mathrm{v}}_{\mathrm{n}}^{*}\right\}=\left\{\left(\max _{\mathrm{j}} \mathrm{v}_{\mathrm{ij}} \mid \mathrm{i} \in \mathrm{B}\right),\left(\min _{\mathrm{j}} \mathrm{v}_{\mathrm{ij}} \mid \mathrm{i} \in \mathrm{C}\right)\right\} \\
& \mathrm{A}^{-}=\left\{\tilde{\mathrm{v}}_{1}^{-}, \tilde{\mathrm{v}}_{2}^{-}, \ldots, \tilde{\mathrm{v}}_{\mathrm{n}}^{-}\right\}=\left\{\left(\min _{\mathrm{j}} \mathrm{v}_{\mathrm{ij}} \mid \mathrm{i} \in \mathrm{B}\right),\left(\max _{\mathrm{j}} \mathrm{v}_{\mathrm{ij}} \mid \mathrm{i} \in \mathrm{C}\right)\right\}
\end{aligned}
$$

Where $\tilde{v}_{i}^{*}$ is the max value of $\mathrm{i}$ for all the alternatives and $\tilde{v}_{1}^{-}$is the min value of $i$ for all the alternatives. $B$ and $C$ represent the positive and negative ideal solutions, respectively.

Step 5 calculate the distance between each alternative and the fuzzy positive ideal solution a+ and the distance between each alternative and the fuzzy negative ideal solution

The distance between each alternative and FPIS and the distance between each alternative and FNIS are respectively calculated as follows:

$$
\begin{aligned}
& \mathrm{S}_{\mathrm{i}}^{*}=\sum_{\mathrm{j}=1}^{\mathrm{n}} \mathrm{d}\left(\tilde{\mathrm{v}}_{\mathrm{ij}}, \tilde{\mathrm{v}}_{\mathrm{j}}^{*}\right) \mathrm{i}=1,2, \ldots, \mathrm{m} \\
& \mathrm{S}_{\mathrm{i}}^{-}=\sum_{\mathrm{j}=\mathrm{i}}^{\mathrm{n}} \mathrm{d}\left(\tilde{\mathrm{v}}_{\mathrm{ij}}, \tilde{\mathrm{v}}_{\mathrm{j}}^{-}\right) \mathrm{i}=1,2, \ldots, \mathrm{m}
\end{aligned}
$$

$\mathrm{d}$ is the distance between two fuzzy numbers, when given two triangular fuzzy numbers $\left(a_{1}, b_{1}, c_{1}\right)$ and $\left(a_{2}\right.$, $\left.b_{2}, c_{2}\right)$, e distance between the two can be calculated as follows:

$$
d_{v}\left(\tilde{M}_{1}, \tilde{M}_{2}\right)=\sqrt{\frac{1}{3}\left[\left(a_{1}-a_{2}\right)^{2}+\left(b_{1}-b_{2}\right)^{2}+\left(c_{1}-c_{2}\right)^{2}\right]}
$$

Note that $d\left(\tilde{v}_{i j}, \tilde{v}_{j}^{*}\right)$ and $d\left(\tilde{v}_{i j}, \tilde{v}_{j}^{-}\right)$are crisp numbers.

\section{Step 6 calculate the closeness coefficient and rank the alternatives}

The closeness coefficient of each alternative can be calculated as follows:

$$
\mathrm{CC}_{\mathrm{i}}=\frac{\mathrm{S}_{\mathrm{i}}^{-}}{\mathrm{S}_{\mathrm{i}}^{+}+\mathrm{S}_{\mathrm{i}}^{-}}
$$

\section{Result}

This section provides a systematic and accurate description of the observational data, their viewpoint as well as the findings to be drawn from the experiment. The alternatives in terms of various criteria are evaluated and the results of the decision matrix are shown as follows. The matrix below represents the arithmetic mean of all experts when multiple experts participate in the evaluation. The following Table 4 shows the decision matrix with respect to criteria and alternatives.

Further the following Table 5 shows the normalized decision matrix.

The following Table 6 demonstrates the weighted normalized decision matrix

Table 5 A normalized decision matrix

\begin{tabular}{llllllll}
\hline & C1 & C2 & C3 & C4 & C5 & C6 & C7 \\
\hline A1 & $0.407,0.640,0.869$ & $0.520,0.742,0.947$ & $0.570,0.785,0.955$ & $0.542,0.758,0.943$ & $0.575,0.792,0.959$ & $0.636,0.842,1.000$ & $0.595,0.814,0.991$ \\
A2 & $0.556,0.790,1.000$ & $0.591,0.809,0.987$ & $0.525,0.749,0.951$ & $0.612,0.833,1.000$ & $0.629,0.842,1.000$ & $0.566,0.772,0.939$ & $0.568,0.795,0.977$ \\
A3 & $0.565,0.794,0.991$ & $0.609,0.827,1.000$ & $0.596,0.816,1.000$ & $0.604,0.815,0.982$ & $0.611,0.824,1.000$ & $0.561,0.768,0.934$ & $0.595,0.814,1.000$ \\
A4 & $0.537,0.771,0.972$ & $0.573,0.787,0.960$ & $0.534,0.753,0.942$ & $0.515,0.731,0.921$ & $0.557,0.769,0.955$ & $0.513,0.732,0.930$ & $0.514,0.741,0.936$ \\
A5 & $0.547,0.780,0.995$ & $0.556,0.773,0.951$ & $0.578,0.798,0.982$ & $0.533,0.749,0.938$ & $0.529,0.756,0.959$ & $0.496,0.715,0.912$ & $0.495,0.723,0.932$ \\
\hline
\end{tabular}


Table 6 The weighted normalized decision matrix

\begin{tabular}{llllllll}
\hline & C1 & C2 & C3 & $C 4$ & $C 5$ & $C 6$ & $C 7$ \\
\hline A1 & $0.058,0.092,0.124$ & $0.074,0.106,0.135$ & $0.081,0.112,0.137$ & $0.077,0.108,0.135$ & $0.082,0.113,0.137$ & $0.091,0.120,0.143$ & $0.085,0.116,0.142$ \\
A2 & $0.080,0.113,0.143$ & $0.085,0.116,0.141$ & $0.075,0.107,0.136$ & $0.088,0.119,0.143$ & $0.090,0.120,0.143$ & $0.081,0.110,0.134$ & $0.081,0.114,0.140$ \\
A3 & $0.081,0.114,0.142$ & $0.087,0.118,0.143$ & $0.085,0.117,0.143$ & $0.086,0.117,0.140$ & $0.087,0.118,0.143$ & $0.080,0.110,0.134$ & $0.085,0.116,0.143$ \\
A4 & $0.077,0.110,0.139$ & $0.082,0.112,0.137$ & $0.076,0.108,0.135$ & $0.074,0.105,0.132$ & $0.080,0.110,0.137$ & $0.073,0.105,0.133$ & $0.073,0.106,0.134$ \\
A5 & $0.078,0.112,0.142$ & $0.079,0.111,0.136$ & $0.083,0.114,0.140$ & $0.076,0.107,0.134$ & $0.076,0.108,0.137$ & $0.071,0.102,0.130$ & $0.071,0.103,0.133$ \\
\hline
\end{tabular}

The positive and negative ideal solutions are shown in the Table 7.

The Table 8 shows distance from positive and negative ideal solutions.

The best alternative is closest to the FPIS and farthest to the FNIS. The closeness coefficient of each alternative and the ranking order of it are shown in the Table 9.

The following Fig. 5 shows the closeness coefficient of each alternative based on the findings of this research study.

The closeness coefficient $(\mathrm{Ci})$ of different alternatives is estimated as $0.438,0.74,0.842,0.322$ and 0.341 for A1, A2, A3, A4 and A5 respectively. The findings show the A3 has highly effective and efficient security requirements engineering approach for trustworthy healthcare software development.

\section{Discussion}

Today's several malicious attempts are by technological flaws in healthcare applications such as SQL injection, session hijacking or cross-site scripting. Healthcare applications which are susceptible to such vulnerabilities have become a convenient target for cyber criminals and therefore most frequently lead to confidential information breaches. The confidentiality of patient's data remains the most significant challenge to reach when contemplating the implementation of Healthcare Information Systems (HIS) in the healthcare sector. Recently, so many studies were performed to recognize potential healthcare security threats, as well as a variety of alternatives were suggested to facilitate security requirements for security-critical healthcare applications. In this study we undertake the powerful fuzzy TOPSIS technique for the selection of effective Security Requirements

Table 7 The positive and negative ideal solutions

\begin{tabular}{lll}
\hline & Positive ideal & Negative ideal \\
\hline C1 & $0.081,0.114,0.143$ & $0.058,0.092,0.124$ \\
C2 & $0.087,0.118,0.143$ & $0.074,0.106,0.135$ \\
C3 & $0.085,0.117,0.143$ & $0.075,0.107,0.135$ \\
C4 & $0.088,0.119,0.143$ & $0.074,0.105,0.132$ \\
C5 & $0.090,0.120,0.143$ & $0.076,0.108,0.137$ \\
C6 & $0.091,0.120,0.143$ & $0.071,0.102,0.130$ \\
C7 & $0.085,0.116,0.143$ & $0.071,0.103,0.133$ \\
\hline
\end{tabular}

Engineering approach to assist the software developers in developing trustworthy healthcare software.

Based on the findings, it is clear that Security Threat Oriented Requirements Engineering (STORE) methodology is the most effective SRE approach based on the security experts' selections. This undoubtedly shows that the recognition of threats is very much significant and every security requirement engineering approach should consider this criterion. In many existing security requirements engineering methods, the threat identification has played a significant role in eliciting effective security requirements for a software project. Prior to eliciting the effective security requirements of a healthcare software system, it is significant to identify all the possible threats to the software system. Assessing the threats to a software system assists software developers to build complete and accurate security requirements [2, 29].

Further risk analysis and stakeholder's involvement in the security requirements engineering process is also important. Although considering stakeholder's view in security requirement engineering is an important concern, but only some security requirements engineering approaches to address this concern. This doesn't mean that it is impossible to consider the views of different stakeholders using other methods. However, most of the security requirements do not capture this issue in their various activities [30-33]. The security requirements engineering approach should propose steps to establish cooperation between different security concerns accepted by different stakeholders. All potential stakeholders must be incorporated during the security requirements engineering process. There are comprehensive security threat models but stakeholder's identification is not emphasized. There is a need to provide steps for considering the security interests of all the stakeholders of the software system and also involves the effective mechanism for threat agent identification and risk analysis for easy, complete, and well-organized security requirements engineering.

After the criteria were prioritized accordingly, the model used in this study enabled us to analyses the main concept of the consistency of preferences made by the security experts. Furthermore, the main purpose of this study is to provide a model enabling security experts to make a more consistent decision for trustworthy 


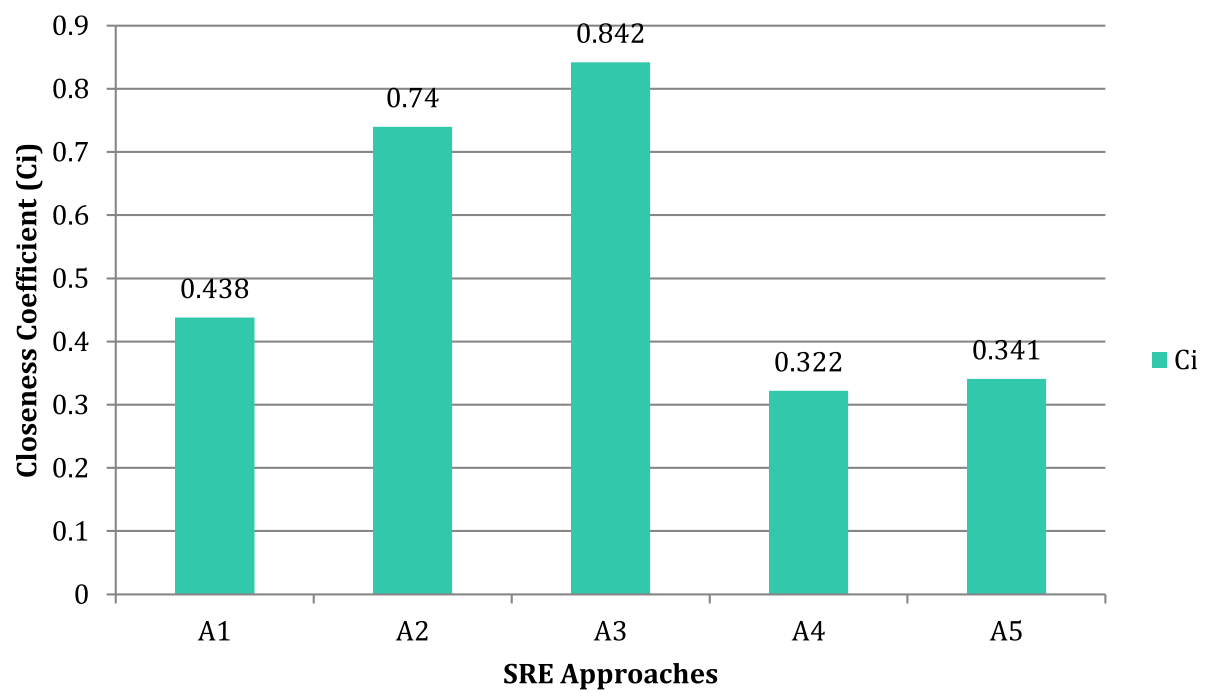

Fig. 5 Closeness coefficient graph

healthcare software development. After the features of the security requirements engineering method became clear, this model can be used to predict effective security requirements engineering approach selection in the real world. This study determined the priority of alternatives that are considered in selecting an effective security requirements engineering method respective of criteria for trustworthy healthcare software development. These criteria highlight the prioritized SRE approaches to which a software developer should pay attention.

\section{Conclusions}

Effective security requirements engineering approach selection decision is essential for the trustworthy healthcare software development. Determining among the many existing SRE methods is a challenging decision-making problem due to the fact that each approach has advantages as well as disadvantages. We implemented the results of a study on the application of fuzzy TOPSIS methodology. A set of ISO 27005 standard criteria identified based on the literature review and organized into a rational hierarchical structure consisting of the seven main

Table 8 Distance from positive and negative ideal solutions

\begin{tabular}{lll}
\hline & Distance from positive ideal & Distance from negative ideal \\
\hline A1 & 0.055 & 0.043 \\
A2 & 0.025 & 0.072 \\
A3 & 0.015 & 0.082 \\
A4 & 0.066 & 0.031 \\
A5 & 0.064 & 0.033 \\
\hline
\end{tabular}

criteria and five alternatives. The consistency ratios were less than 0.10 for all the 25 security experts in collected form responses. The research findings suggest that the STORE approach (with $\mathrm{Ci}$ value 0.842 ) is more effective than SQUARE (0.74), SREP (0.438), SREF (0.341), and MOSRE (0.322) in manipulating performance towards security requirements engineering approaches. Determining weights of essential motivation, purpose, and consciousness focus areas can help security decision-making and compliance with policy, and support design of effective security requirements engineering. However, these weights may in turn be affected by local organizational and educational factors. The presented fuzzy TOPSIS results in this paper can be used to select or design an effective security requirements engineering approach that may assist the software developers in developing a trustworthy healthcare software system. Several other fuzzy decision-making approaches are available like VIKOR, fuzzy ANP, PROMETHE, and many others can be used for future research, and their findings can be compared with the findings obtained in this study. The outcome discussed in this research may be used by the software professionals working in

Table 9 Closeness coefficient

\begin{tabular}{lll}
\hline & $\mathrm{Ci}$ & Rank \\
\hline A1 & 0.438 & 3 \\
A2 & 0.74 & 2 \\
A3 & 0.842 & 1 \\
A4 & 0.322 & 5 \\
A5 & 0.341 & 4 \\
\hline
\end{tabular}


the clinical, education, and healthcare activities related to software development.

\section{Abbreviations}

MCDM: Multi-criteria decision making; MADM: Multiple Attribute Decisionmaking; NIS: Negative ideal solution; PIS: Positive ideal solution; TOPSIS: Technique for order preference by similarity to ideal solution; SRE: Security Requirements Engineering

\section{Acknowledgments}

Authors wish to thank all contributing experts. Authors would also like to thank two reviewers and editor for their insightful and helpful comments and suggestions that have led to significant improvements in the paper.

\section{Authors' contributions}

MTJA conceived and planned the experiments, implemented the experiments, examined the data, contributed reagents/materials/analysis tools, authored or reviewed drafts of the paper, approved the final draft. FAA helped in theoretical development of the manuscript, approved the final draft. DP conceived and designed the experiments, performed the experiments, contributed reagents/materials/analysis tools, prepared figures and/or tables, performed the computation work, approved the final draft. AA analyzed the data, contributed analysis tools, authored or reviewed drafts of the paper, approved the final draft. All authors approved the final version of the manuscript.

\section{Funding}

Funding for this work was provided by grant number 12-INF2970-10 from the National Science, Technology and Innovation Plan (MAARIFAH), the King Abdul-Aziz City for Science and Technology (KACST), Kingdom of Saudi Arabia. We thank the Science and Technology Unit at Umm Al-Qura University for their continued support.

\section{Availability of data and materials}

All data generated or analysed during this study are included in this paper.

\section{Ethics approval and consent to participate}

Informed consent was obtained from all participants prior to the survey.

\section{Consent for publication}

Not applicable.

\section{Competing interests}

The authors declare that they have no competing interests.

\section{Author details}

'Department of Information Technology, Babasaheb Bhimrao Ambedkar University, Lucknow, Uttar Pradesh, India. ${ }^{2}$ Department of Computer Engineering, College of Computer and Information Systems, Umm Al-Qura University, Mecca, Saudi Arabia.

Received: 5 February 2020 Accepted: 3 August 2020

Published online: 18 September 2020

\section{References}

1. ur Rehman S, Gruhn V. Recommended architecture for car parking management system based on cyber-physical system. In: 2017 International Conference on Engineering \& MIS (ICEMIS): IEEE; 2017. p. 1-6.

2. Ansari MTJ, Pandey D, Alenezi M. STORE: security threat oriented requirements engineering methodology: Journal of King Saud UniversityComputer and Information Sciences; 2018.

3. Manadhata PK, Karabulut Y, Wing JM. Report: measuring the attack surfaces of enterprise software. In International Symposium on Engineering Secure Software and Systems. Berlin, Heidelberg: Springer; 2009. p. 91-100.

4. Pattakou A, Kalloniatis C, Gritzalis S. Security and privacy requirements engineering methods for traditional and cloud-based systems: a review. Cloud Comput. 2017;2017:155.

5. Ansari MTJ, Pandey D. Risks, security, and privacy for HIV/AIDS data: big data perspective. In: Censorship, Surveillance, and Privacy: Concepts, Methodologies, Tools, and Applications: IGl global; 2019. p. 58-74.
6. Healthcare Data Breach Statistics. (2020, February 18). Retrieved June 15, 2020, from https://www.hipaajournal.com/healthcare-data-breach-statistics/.

7. McGraw G. Security fatigue? Shift your paradigm. Computer. 2014;47(3):813.

8. Muñante D, Chiprianov V, Gallon L, Aniorté P. A review of security requirements engineering methods with respect to risk analysis and modeldriven engineering. In International Conference on Availability, Reliability, and Security. Cham: Springer; 2014. p. 79-93.

9. IDC, Asia/Pacific* Spending on Security Solutions Projected to Reach USD 16.4 Billion in 2019, IDC Reports. (2019, November 18). Retrieved January 1, 2020, from https://www.idc.com/getdoc.jsp?containerld=prAP45656119.

10. Pandey $D$, Suman $U$, Ramani AK. Security requirement engineering issues in risk management. Int J Comput Appl. 2011;975:8887.

11. Hwang CL, Yoon K. Methods for multiple attribute decision making. In: multiple attribute decision making. Berlin, Heidelberg: Springer; 1981. p. 58191.

12. Mellado D, Blanco C, Sánchez LE, Fernández-Medina E. A systematic review of security requirements engineering. Comput Stand Interfaces. 2010;32(4): $153-65$.

13. Devanbu PT, Stubblebine S. Software engineering for security: a roadmap. In: Proceedings of the Conference on the Future of Software Engineering; 2000. p. 227-39. ACM.

14. Lee $Y$, Lee J, Lee $Z$. Integrating software lifecycle process standards with security engineering. Comput Secur. 2002;21(4):345-55.

15. Kotonya G, Sommerville I. Requirements engineering: processes and techniques: Wiley Publishing; 1998.

16. Deshmukh M. Security requirements engineering process. In: Seminar in Information System, Security Engineering; 2009.

17. Gutgarts PB, Temin A. Security-critical versus safety-critical software. In: 2010 IEEE International Conference on Technologies for Homeland Security (HST): IEEE; 2010. p. 507-11.

18. Salini P, Kanmani S. Model oriented security requirements engineering (MOSRE) framework for web applications. In: Advances in Computing and Information Technology. Berlin, Heidelberg: Springer; 2013. p. 341-53.

19. Haley C, Laney R, Moffett J, Nuseibeh B. Security requirements engineering: a framework for representation and analysis. IEEE Trans Softw Eng. 2008; 34(1):133-53.

20. Mellado D, Fernández-Medina E, Piattini M. Applying a security requirements engineering process. In: European Symposium on Research in Computer Security. Berlin, Heidelberg: Springer; 2006. p. 192-206.

21. Mead, N. R., \& Stehney, T. (2005). Security quality requirements engineering (SQUARE) methodology (Vol. 30, no. 4, pp. 1-7). ACM

22. Bulusu ST, Laborde R, Wazan AS, Barrere F, Benzekri A. Applying a requirement engineering based approach to evaluate the security requirements engineering methodologies. In: Proceedings of the 33rd Annual ACM Symposium on Applied Computing: ACM; 2018. p. 1316-8.

23. Herrmann DS. Using the common criteria for IT security evaluation: CRC Press; 2002.

24. Kou G, Lu Y, Peng Y, Shi Y. Evaluation of classification algorithms using MCDM and rank correlation. Int J Inf Technol Decis Mak. 2012;11(01):197225.

25. Kou G, Peng Y, Wang G. Evaluation of clustering algorithms for financial risk analysis using MCDM methods. Inf Sci. 2014;275:1-12.

26. Kuruoglu E, Guldal D, Mevsim V, Gunvar T. Which family physician should I choose? The analytic hierarchy process approach for ranking of criteria in the selection of a family physician. BMC Med Inform Decis Making. 2015; 15(1):63.

27. Ashrafzadeh M, Rafiei FM, Isfahani NM, Zare Z. Application of fuzzy TOPSIS method for the selection of warehouse location: a case study. Interdiscip J Contemp Res Business. 2012;3(9):655-71.

28. Sevkli, M., Zaim, S., Turkyilmaz, A., \& Satir, M. (2010). An application of fuzzy Topsis method for supplier selection. In International Conference on Fuzzy Systems (pp. 1-7). IEEE.

29. Myagmar S, Lee AJ, Yurcik W. Threat modeling as a basis for security requirements. In: Symposium on requirements engineering for information security (SREIS), vol. 2005; 2005. p. 1-8.

30. Ramachandran M. Software security requirements management as an emerging cloud computing service. Int J Inf Manag. 2016;36(4):580-90.

31. Fabian B, Gürses S, Heisel M, Santen T, Schmidt H. A comparison of security requirements engineering methods. Requir Eng. 2010;15(1):7-40. 
32. Ullah S, lqbal M, Khan AM. A survey on issues in non-functional requirements elicitation. In: International Conference on Computer Networks and Information Technology: IEEE; 2011. p. 333-40.

33. Luburić, N., Sladić, G., \& Milosavljević, B. (2018). Applicability issues in security requirements engineering for agile development. In Proceedings/8 th International conference on applied internet and information technologies (Vol. 8, no. 1, pp. II-VII). "St Kliment Ohridski" university-Bitola, Faculty of Information and Communication Technologies-Bitola, republic of Macedonia.

\section{Publisher's Note}

Springer Nature remains neutral with regard to jurisdictional claims in published maps and institutional affiliations.

Ready to submit your research? Choose BMC and benefit from:

- fast, convenient online submission

- thorough peer review by experienced researchers in your field

- rapid publication on acceptance

- support for research data, including large and complex data types

- gold Open Access which fosters wider collaboration and increased citations

- maximum visibility for your research: over $100 \mathrm{M}$ website views per year

At $\mathrm{BMC}$, research is always in progress.

Learn more biomedcentral.com/submissions 